\title{
PET-Saúde: formando discentes multiplicadores - relato de experiência
}

\author{
Késsia Nara Andrade Sales*, Fabiana Angélica de Paula**, Mirtes Ribeiro***, Liliane da \\ Consolação Campos Ribeiro****, Sylvana Mara Canuto*****
}

\author{
* Discente do curso de Graduação em Odontologia da UFVJM. \\ Bolsistas do PET-Saúde/Saúde Mental-Drogas/UFVJM \\ ** Professora Auxiliar I do Departamento de Enfermagem da UFVJM. \\ Mestranda do PRPPG/UFVJM. Tutora do PET-Saúde/Saúde \\ Mental/UFVJM \\ *** Professora Assistente do Departamento de Enfermagem da UFVJM. \\ Doutoranda do Programa Saúde da Criança e do Adolescente da \\ Faculdade de Medicina da UFMG. Coordenadora dos PET-Saúde/ \\ Saúde Mental e Vigilância à Saúde/UFVJM \\ **** Professora Assistente do Departamento de Enfermagem da \\ UFVJM. Doutoranda do Programa Saúde da Criança e do \\ Adolescente da Faculdade de Medicina da UFMG. Tutora do \\ PET-Saúde/VS \\ ***** Psicóloga do Centro de Assistência e Promoção Social/Diamantina. \\ Preceptora do PET-Saúde/Saúde Mental/UFVJM
}

\section{RESUMO}

A interação dos profissionais da saúde ainda na graduação, torna-se possível por meio de iniciativas como o Programa de Educação pelo Trabalho para a Saúde (PET-Saúde), o qual se constitui em um instrumento que viabiliza a inserção dos estudantes no Sistema Único de Saúde - SUS, visando à iniciação ao trabalho científico, estágios e vivências do cotidiano do serviço, contribuindo para a formação de profissionais mais comprometidos com a realidade de saúde e sua transformação. O presente trabalho trata-se de um relato de experiência de caráter descritivo, com o objetivo de relatar a experiência de uma aluna de graduação em Odontologia da Universidade Federal dos Vales do Jequitinhonha e Mucuri (UFVJM), participante do PET-Saúde/Saúde Mental-Drogas/ UFVJM e ex-participante do PET-Saúde/Vigilância em Saúde/UFVJM. O PET-Saúde/Vigilância a Saúde da UFVJM, é composto por dois grupos tutoriais sendo que o grupo do qual a acadêmica participou visava ampliar o acesso á testagem para diagnóstico de contaminação pelo vírus HIV, utilizando testes-rápidos. O PET-Saúde/Saúde Mental-Drogas/UFVJM tem como proposta, dentre outros objetivos, qualificar acadêmicos técnicamente, científicamente e tec- nologicamente na área da saúde mental, com abordagem a álcool e outras drogas. Além disso, o programa PET-Saúde/UFVJM estimula o aluno a desenvolver atividades de pesquisa com propostas de mudança efetivas em sua realidade. Desta forma, percebe-se que a integração ensino-serviço-comunidade pode ser utilizada como um instrumento valioso na promoção social e no exercício da cidadania, tornando-se fundamental na diminuição das problemáticas sociais existentes.

\section{DESCRITORES}

AIDS. Drogas. PET-Saúde/UFVJM. Odontologia. Relato de experiência.

$\Delta$ interação dos profissionais da saúde torna-se possível por meio de iniciativas como o Programa de Educação pelo Trabalho para a Saúde (PET-Saúde), o qual se constitui em um instrumento que viabiliza a inserção dos estudantes no Sistema Único de Saúde - SUS ainda na graduação, visando à iniciação ao trabalho científico, estágios e vivências na realidade do serviço. Promove uma articulação entre ensinoserviço-comunidade por meio da formação de uma equipe interdisciplinar, composta por acadêmicos e 
profissionais da saúde, caracterizando-se como instrumento para qualificação em serviço, enriquecendo o conhecimento acadêmico e contribuindo para a formação de profissionais mais comprometidos com a realidade de saúde e sua transformação. ${ }^{2}$

O PET-Saúde/Vigilância a Saúde (PET-Saúde/ VS) da Universidade Federal dos Vales do Jequitinhonha e Mucuri (UFVJM) têm como objetivos proporcionar a formação de profissionais capacitados e engajados com os serviços de atenção á saúde, com foco na vigilância à saúde, pautados no senso crítico, na cidadania e na função social da educação, por meio das atividades de ensino, pesquisa e extensão e proporcionar a melhoria da qualidade dos serviços de vigilância á saúde prestados á população, por meio da permuta de metodologias e tecnologias oriundas dos serviços de Vigilância a Saúde e da Instituição de Educação Superior. Para a implementação desta proposta inicialmente foram constituídos dois grupos tutoriais, um cuja meta é a Vigilância das Doenças Diarréicas Agudas e das Doenças Transmitidas por Alimentos e outro cuja proposta era de ampliar o acesso á testagem para diagnóstico de contaminação pelo vírus HIV, utilizando testes-rápidos. Ambos tendo com cenários de práticas os 34 municípios da jurisdição da Superintendência Regional de Saúde de Diamantina - MG.

A Síndrome da Imunodeficiência Adquirida (AIDS) é, atualmente, uma epidemia que tem avançado progressivamente em todo o mundo, ultrapassando barreiras geopolíticas, sendo assim o diagnóstico precoce do HIV/AIDS é fator importante para assegurar maior qualidade de vida às pessoas infectadas. Sendo assim, existe no mercado um sistema de teste Rápido HIV que utiliza antígenos fixados para a detecção de anticorpos para o HIV em soro, plasma ou sangue. ${ }^{9} \mathrm{O}$ Teste Rápido é um teste imunocromatográfico rápido de simples execução, além de seguro e seu resultado confiável e sigiloso. Justifica-se então a existência de iniciativas como PETSaúde/VS-HIV/AIDS na perspectiva de promover ações de prevenção e promoção da saúde, bem como o diagnóstico e tratamento precoce da infecção pelo vírus HIV.

O PET-Saúde/Saúde Mental-Drogas/UFVJM tem como proposta, dentre outros objetivos, qualificar acadêmicos técnicamente, científicamente e tecnológicamente na área da saúde mental, com abordagem a álcool e outras drogas.

A dependência de drogas é um problema de destaque social. É considerada um distúrbio, e por isso deve receber um enfoque mais amplo, envolvendo cuidados especiais por parte dos profissionais de saúde. $\mathrm{O}$ uso de substâncias químicas vem aumentando gradativamente, fragilizando a família e o próprio indivíduo, que soma para si uma série de alterações físicas, químicas e emocionais.

A pertinência deste relato está no imenso conhecimento adquirido pelos integrantes do grupo, por meio das pesquisas e das interações profissionais, complementando a formação acadêmica-profissional por meio da interdisciplinaridade, visto que o profissional interdisciplinar deve estar disposto a vivenciar uma prática de constante mudança, não tendo como objetivo único uma meta pré-estabelecida.

Objetiva-se com este, relatar a experiência de uma aluna de graduação em Odontologia da UFVJM, participante do PET-Saúde/Saúde Mental-Drogas/ UFVJM e ex-participante do PET-Saúde/Vigilância em Saúde/UFVJM.

\section{DESCRIÇÃO DA EXPERIÊNCIA}

O presente trabalho trata-se de um relato de experiência de caráter descritivo. A duração da experiência foi de aproximadamente um ano.

A metodologia de trabalho utilizada em ambos os PET-Saúde, trata-se da Técnica de Metodologia ativa do Processo de Ensino Aprendizado, composta das seguintes fases:

- vivência da prática;

- reflexão da prática vivenciada;

- investigação qualificada de informações e

- reflexão da prática com a intenção de transformá-la.

Já quanto ao envolvimento de outros profissionais e instituições, são seguidas as seguintes etapas:

- sensibilização e envolvimento das instituições,

- fortalecimento da ideia de um novo modelo de vigilância e promoção à saúde,

- fortalecimento e desenvolvimento dos profissionais envolvidos nas ações e por fim,

- construção e aplicação do saber aprendido.

Enquanto membro da equipe PET-Saúde/VS, foram desenvolvidas atividades de pesquisa e extensão com enfoque na prevenção e diagnóstico precoce do HIV/AIDS, juntamente com outros discentes dos cursos de Farmácia, Odontologia, Educação Física, Ciências Biológicas e Enfermagem, sob orientação de profissionais (tutores e preceptores) da enfermagem, da odontologia e da fisioterapia. Foram realizadas diversas capacitações que abordavam a epidemiologia 
do HIV/AIDS, bem como os grupos vulneráveis, sintomas, métodos de prevenção e tratamento, mecânica de realização do teste - rápido. Os acadêmicos puderam conhecer a realidade do Centro de Testagem e Aconselhamento (CTA) da cidade de Diamantina. Foi possível ainda desenvolver atividades de extensão como palestras sobre HIV/AIDS para diferentes públicos, caminhadas de conscientização, distribuição de preservativos e material informativo sobre o tema.

O PET-Saúde/Saúde Mental-Drogas/UFVJM é composto por acadêmicos dos cursos de Enfermagem, Fisioterapia, Nutrição, Farmácia e Odontologia, orientados por profissionais (preceptores e tutores) das áreas de enfermagem, psicologia e assistência social. Neste houve cursos de capacitação, dividido em dois módulos, sendo que no Módulo I abordou assuntos a cerca da saúde mental e suas complexidades, reforma psiquiátrica, organização dos serviços de saúde mental. No Módulo II foram discorridos temas como:

- transtornos decorrentes do uso de drogas e álcool,

- abordagem e tratamento,

- a dependência química,

- legislação da política nacional de combate ao uso de álcool/drogas e saúde mental/dependência química.

A fim de cumprir os objetivos propostos pelo projeto, a equipe foi dividida em três grupos, cada qual com uma meta distinta:

- construção do Diagnóstico Administrativo e Situacional do Centro de Assistência e Promoção Social (CAPS) Renascer/Diamantina;

- capacitar os dispositivos comunitários de álcool e drogas existentes na cidade e

- capacitar os profissionais das ESFs (Estratégia Saúde da família) a cerca do tema saúde mental e drogas.

Sendo proposto aos acadêmicos mudarem de grupo periodicamente e desenvolver continuadamente o trabalho iniciado pelos colegas, oportunizando a estes a vivencia enquanto acadêmicos para a aquisição de habilidades necessárias as experiências para o bom andamento do serviço de saúde, apesar da grande rotatividade profissional.

\section{RESULTADOS E DISCUSSÃO}

A interdisciplinaridade implica uma consciência dos limites e das potencialidades de cada campo de saber para que possa haver uma abertura em direção de um fazer coletivo. $\mathrm{O}$ trabalho em equipe tem como compromisso a geração de dispositivos renovados para o trabalho, sendo necessário que cada profissional se familiarize com as outras áreas, de modo legitimado e em relações horizontais. ${ }^{6}$

Por ser composto por uma equipe multi e interdisciplinar, o PET-Saúde possibilita ao discente conhecer melhor a dinâmica de funcionamento do trabalho em equipe dos serviços de saúde pública, entendendo a interdependência positiva que existe entre os diferentes profissionais bem como a aproximação com o contexto social da população a ser assistida, além de realizar uma articulação entre os diversos profissionais de saúde que compõe a rede de assistência.

O programa estimula o aluno a desenvolver atividades de pesquisa, investigação científica, coleta, tabulação de dados e análises críticas com propostas de mudança efetivas em uma determinada realidade. Para atingir tal proposta são realizadas capacitações sobre os portais de pesquisa, programas estatísticos, geoprocessamento, revisão integrativa, dentre outros.

A fim de discutir a ampliação do acesso ao diagnóstico da infecção pelo HIV, em atendimento aos princípios da equidade e da integralidade da assistência, bem como da universalidade de acesso aos serviços de saúde do SUS, o grupo PET-Saúde/VS-HIV/ AIDS em parceria com a Coordenação Regional de DST/HIV/AIDS e o Programa Municipal de DST/ HIV/AIDS de Diamantina realizou um encontro intitulado "Estratégias para sensibilização quanto à necessidade de testagem para detecção de portadores do HIV na região do Vale do Jequitinhonha".

Foi abordado no encontro temas como a utilização do teste rápido, a importância do aconselhamento pré e pós-teste e a realização do mesmo, além de propor atividades de mobilização da comunidade e a presença do CTA Itinerante nos municípios participantes e que se manifestarem interesse por tal. Foram elaborados também planos para a identificação da população vulnerável dos municípios e estratégias para concientizá-las a cerca da prevenção da contaminação pelo vírus HIV/AIDS bem como a importância do diagnóstico precoce.

Teve duração de 16 horas e contou com a participação de profissionais ligados à epidemiologia dos municípios da macrorregional de Diamantina. Foi muito valiosa a experiência adquirida pelos petianos em tal evento, uma vez lhes possibilitou conhecer a realidade de vários municípios do Vale Jequitinho- 
nha, e também a troca de vivências entre vários profissionais da saúde participantes do mesmo.

$\mathrm{O}$ dia $1^{\circ}$ de dezembro é marcado pelo Dia Mundial de Luta Contra a AIDS. Para lembrar a data, a prefeitura de Diamantina realizou diversas ações voltadas para o combate à discriminação e o preconceito, tal como uma caminhada pela vida nas ruas da cidade, evento do qual o grupo PET-Saúde/VS participou como convidado e também contribuiu na distribuição de preservativos e material informativo.

A campanha nacional do referido ano (2010) teve como foco principal a conscientização de homens e mulheres com idade entre 14 e 24 anos, diante do preconceito. Tinha proposta de dar maior visibilidade às questões de viver com HIV/AIDS, além de combater o estigma e a discriminação, enfatizando a proximidade da doença com o universo jovem. A fim de atingir este público alvo a caminhada teve como encerramento com um show com uma banda local, a Batcaverna, no qual os vocalistas da banda falavam sobre o tema durante a sua apresentação. O evento reuniu aproximadamente 8 mil pessoas.

Previamente à campanha foi realizada uma oficina intitulada "BAT-papo" com a participação dos integrantes da banda, a fim de os tornarem multiplicadores e cidadãos parceiros das ações de educação em saúde, já que são admirados e vistos como referência para a juventude. Discutiu-se temas como o uso da camisinha, a campanha ministerial "Fique Sabendo" e os termos vulnerabilidade e comportamentos de risco, num clima informal e descontraído.

Devido ao aumento do índice de pessoas contaminadas pelo vírus HIV, percebe-se a necessidade de campanhas que conscientizem sobre o uso de preservativos e sobre a importância dos testes rápidos para detecção precoce do vírus. Com o objetivo de abordar realidade dentro da UFVJM, foi realizada a Campanha "Universidade - Fique Sabendo", uma inciativa do PET-Saúde/VS/UFVJM em parceria com a Secretaria Estadual de Saúde (SES/MG) e a Superintendência Regional de Saúde de Diamantina (SRS-D).

A campanha aconteceu em dois dias nos diferentes campus da universidade e teve como foco a divulgação do teste rápido para a sorologia do HIV/AIDS e da rede de serviços disponíveis, bem como campanhas de prevenção e conscientização, e ainda encaminhar as pessoas diagnosticadas com HIV aos serviços especializados. Foram distribuídos preservativos e materiais informativos sobre HIV/AIDS, realizados aconselhamentos pré-teste de forma coletiva, testagem dos universitários e funcionários interessados, utilizando testes rápidos para o HIV, e aconselhamento individual pós-teste, possibilitando o esclarecimento de dúvidas em relação ao teste e à doença.

A campanha teve muito sucesso, uma vez que o número de acadêmicos atingidos superou as expectativas, sendo que estes relatarem se sentir muito mais "à vontade" conversar sobre um assunto tão polêmico que é o HIV/AIDS com pessoas que vivem a mesma realidade que eles, também por atingir o objetivo de ampliar o acesso à testagem dos universitários da UFVJM, para diagnóstico de contaminação pelo vírus HIV, utilizando testes-rápidos de forma gratuita e sigilosa.

No decorrer do projeto, petianos e a tutora ministraram uma palestra com o tema "DST e AIDS" na "I Semana Interna de Prevenção de Acidentes no Trabalho-SIPAT". O público alvo foi de aproximadamente 70 trabalhadores da Construtora responsável pela ampliação do Campus da Universidade, sendo estes do sexo masculino, na faixa etária de 18 a 60 anos de idade. Foi um desafio para as acadêmicas, mas também foi bastante exitoso, pois apesar das dificuldades de 2 alunas falarem de um tema tão polêmico para um público composto apenas de homens de uma faixa etária tão ampla, conseguiram desenvolve-lo com habilidade.

Com os conhecimentos adquiridos no PET-Saúde/VS foi possível também orientar colegas de curso a cerca de uma conduta correta após acidentes com material biológico na clinica odontológica, bem como uma conscientização em relação à postura de trabalho na prevenção da contaminação ocupacional e também a contaminação cruzada.

A experiência adquirida com o PET-Saúde/Vigilância em Saúde possibilita enquanto graduanda em odontologia uma formação diferenciada, habilitando-a ao trato de pacientes com doenças infecto-contagiosas. O odontólogo tem um papel relevante frente ao diagnostico precoce da contaminação pelo HIV, uma vez que $90 \%$ dos pacientes infectados pelo vírus apresentam, a qualquer momento durante o curso da infecção, manifestações bucais, podendo ser ele ainda o primeiro a detectar e diagnosticar lesões indicadoras da Aids. ${ }^{3,7}$

Embora a microbiota de um portador de HIV/ AIDS seja semelhante à aquela encontrada em soronegativos, ela pode ser acrescida por microrganismos oportunistas. ${ }^{7}$ Para tanto, o profissional deve estar treinado e capacitado sobre as intercorrências dessas patologias, sabendo diagnosticá-las e tratá-las a contento, e também estar preparado a orientar o seu 
paciente para a necessidade imediata de uma avaliação médica, contribuindo assim para uma intervenção precoce.

O PET-Saúde/Saúde Mental-Drogas/UFVJM possibilita ao acadêmico a conhecer o funcionamento do CAPS e dos dispositivos de proteção contra álcool e outras drogas (alcoólicos anônimos, narcóticos anônimos, dentre outros).

É crescente o número de indivíduos dependentes químicos, bem como a falta de aprofundamento nesse assunto por parte da Odontologia. No entanto muitas vezes os profissionais dessa área são os primeiros a terem a oportunidade de diagnosticar o aparecimento de possíveis alterações surgidas em virtude do consumo de cigarro, álcool ou drogas ilícitas. ${ }^{4,8}$

O drogadito apresenta uma perda da auto-estima, levando a um descuido com a saúde geral e bucal. A droga pode ser fator etiológico de inúmeras lesões bucais, pois pode acarretar uma diminuição no fluxo salivar, desgaste dental, perda óssea, aumento do CPOD (dentes cariados, perdidos e obturados), problemas periodontais, xerostomia, bruxismo, estomatites, hipoestesia e dor, além da ocorrência de leucoplasias e carcinomas. ${ }^{4,5,8} \mathrm{O}$ consumo excessivo de drogas promove mudanças efetivas em células da mucosa bucal, facilitando a penetração de carcinógenos. ${ }^{1}$

Algumas drogas, como por exemplo, a maconha, afetam a produção de células de defesa, levando a um quadro de imunossupressão, deixando o indivíduo mais vulnerável a infecções oportunistas, tais como a candidíase. Pode ocorrer também necrose tecidual devido ao déficit de suprimento sanguíneo ocasionado pelo poder vasoconstritor de drogas como a cocaína. ${ }^{4}$

Logo, os conhecimentos adquiridos no PET-Saúde/Saúde Mental-Drogas possibilita a formação de um profissional diferenciado, capaz de reconhecer e entender as alterações que o álcool e outras drogas causam na cavidade bucal. Ele se torna apto em tratar não só a patologia, mas também entender o paciente, informar, de maneira clara e objetiva, todos os prejuízos causados pela droga, na boca e na saúde geral, e motivar ao abandono do vício, fornecendo ferramentas de ajuda para tal. O embasamento teórico-prático torna-o capaz de orientar os pacientes sobre os dispositivos comunitários e a procura de serviços de saúde, tais como o CAPS álcool e drogas.

\section{CONCLUSÃO}

A relevância da experiência se dá pelo fato da in- serção do estudante no cenário vivo de aprendizado ser de grande significado para os processos formadores dos cursos de graduação em saúde, em especial no curso de Odontologia, que muitas vezes não oferece este espaço no currículo oficial.

A possibilidade de troca de saberes entre os integrantes das equipes é outro fator que contribui para a formação dos acadêmicos envolvidos.

Contudo percebe-se que a integração ensino-serviço-comunidade pode ser utilizada como um instrumento valioso na promoção social e no exercício da cidadania, tornando-se fundamental na diminuição das problemáticas sociais existentes.

A Odontologia, além de aliviar a dor, pode contribuir para a reabilitação desses pacientes, auxiliando no desenvolvimento da auto-estima e ampliando a interação social, já que a recuperação implica o resgatar do ser humano em todos os aspectos, seja eliminando o significado psicológico das drogas ou por estar apto a realizar o acolhimento e aconselhamento do soropositivo, bem como orientá-los na busca de serviços especializados, dando ênfase às ações de prevenção e promoção da saúde a fim de proporcioná-lo condições necessárias para manter sua qualidade de vida.

\section{ABSTRACT}

PET-Health: preparing college student facilitators report of an experience

The interaction of health professionals still pursuing undergraduate studies is made possible through initiatives like the Education Program through Health Work (PET-Health). This program acts as an instrument through which students may be included in the Unified Health System (SUS), an inclusion that aims at introducing them to scientific work, internships and daily experience in extending professional services. All this contributes to the training of professionals committed to the reality of the healthcare area and to changing this reality. The present study reports an experience by an undergraduate dental student from the Federal University of the Jequitinhonha and Mucuri Valleys (UFVJM). Said student also participates in the PET / Mental Health-Drugs / UFVJM, and has participated in PET-Health / Health Surveillance / UFVJM. The PET-Health / Health Surveillance of UFVJM consists of two tutorial groups, in that the group in which the student participated aimed at gaining greater access to testing for the diagnosis of HIV infection through a rapid-testing technique. Among other goals, the PET-Health / Mental Health- 
Drugs / UFVJM has the purpose of qualifying students technically, scientifically and technologically in the area of mental health, through an approach focusing on alcohol and other drugs. In addition, the PETHealth / UFVJM program encourages students to develop research activities that propose effective changes in his reality. Thus, it can be concluded that the integration of teaching and community service can be used as a valuable tool in social promotion and exercise of citizenship, and that it is instrumental in reducing the existing social problems.

\section{DESCRIPTORS}

AIDS. Drugs. PET-Saúde/UFVJM. Dentistry. Report of experience.

\section{REFERÊNCIAS}

1. Batista AB, Ferreira FM, Ignácio SA, Machado MAN, Lima AAS. Efeito do tabagismo na mucosa bucal de indivíduos jovens: análise citomorfométrica. Rev Bras Cancerol. 2008;54(1):5-10.

2. Brasil. Portal da Saúde. Programa de Educação pelo Trabalho para a Saúde (PET-Saúde). Brasília: Ministério da Saúde; 2011.

3. Cavassani VGS, SobrinhoJA, Homem MGN, Rapoport. A. Candidíase oral como marcador de prognóstico em pacientes portadores do HIV. Rev Bras Otorrinolaringol. 2002;68(5):630-4.
4. Colodel EV; Silva ELFM; Zielak, JC; Zaitter, W;Crosato EM; Pizzatto E. Alterações bucais presentes em dependentes químicos. Revista Sul-Brasileira de Odontologia. 2009;6(1):44-48.

5. Fernandes JP, Brandão VSG, Lima AAS. Prevalência de lesões cancerizáveis bucais em indivíduos portadores de alcoolismo. Revista Brasileira de Cancerologia. 2008;54(3):239-244

6. Garcia MAA, Pinto ATBCS, Odoni APC, Longhi BS, Machado LI, Linek MDS, Costa NA. Interdisciplinaridade e integralidade no ensino em saúde. Rev. Ciênc. Méd. 2006;15(6):473-485

7. Gasparin AB, Ferreira FV, Danesi CC, Sassi RAM, Silveira J, Martinez AMB, Zhang L, Cesar J. Prevalência e fatores associados às manifestações bucais em pacientes HIV positivos atendidos em cidade sul-brasileira. Cad. Saúde Pública. 2009; 25(6):1307-1315

8. Ribeiro EDP, Oliveira JA, Zambolin AP, Lauris JRP, Tomita NE. Abordagem integrada da saúde bucal de droga-dependentes em processo de recuperação. Pesqui. Odontol. Bras. 2002;16(3):239-245.

9. Silva JAS, Val LF, Nichiata LYI. A estratégia saúde da família e a vulnerabilidade programática na atenção ao HIV/AIDS: uma revisão da literatura. O Mundo da Saúde, São Paulo: 2010;34(1):103-108. 